\title{
Field emission properties of gallium oxide micro- and nanostructures in the scanning electron microscope
}

\author{
Iñaki López, Emilio Nogales, Pedro Hidalgo, Bianchi Méndez*, and Javier Piqueras \\ Facultad de Ciencias Físicas, Departamento de Física de Materiales, Universidad Complutense de Madrid, 28040 Madrid, Spain
}

Received 12 July 2011, revised 14 September 2011, accepted 21 September 2011

Published online 17 October 2011

Keywords doping, field emission, gallium oxide, nanowires

*Corresponding author: e-mail bianchi@fis.ucm.es, Phone: +34 913944 746, Fax: +34 913944547

The field emission properties of gallium oxide nanowires grown by thermal evaporation-deposition have been investigated inside the chamber of a scanning electron microscope. Turn on electric fields and enhancement factors have been determined for Sn doped nanowires. X-ray photoelectron spectroscopy measurements have been performed to calculate the work function of $\mathrm{Sn}$ doped $\mathrm{Ga}_{2} \mathrm{O}_{3}$. The results show improved field emission properties of $\mathrm{Sn}$ doped $\mathrm{Ga}_{2} \mathrm{O}_{3}$ nanowires, with a lower threshold field (below $1.0 \mathrm{~V} / \mu \mathrm{m}$ ). The obtained values are competitive with those achieved in other nanostructured materials, including carbon nanotubes.
1 Introduction Metal oxide nanowires are versatile materials due to their diverse properties and functionalities with applications in multidisciplinary fields. One of the potential applications of these nanowires is its use as field emitters since their particular geometry contributes to lower the threshold electric field for electron emission. Transitionmetal oxide nanowires, such as $\mathrm{MoO}_{3}, \mathrm{WO}_{3}$ or $\mathrm{RuO}_{2}$ [1-4] and transparent conductive oxides, as $\mathrm{ZnO}$ or $\operatorname{In}_{2} \mathrm{O}_{3}[5,6]$ have been demonstrated to show good field emission properties. Among conductive oxides, $\beta-\mathrm{Ga}_{2} \mathrm{O}_{3}$ is a wide band gap semiconductor with optical and electrical properties of interest. For example, tunable light emission by means of doping with optically active ions, e.g. $\mathrm{Cr}, \mathrm{Er}$ and $\mathrm{Eu}$, has been demonstrated in $\mathrm{Ga}_{2} \mathrm{O}_{3}$ nanowires [7-9]. On the other hand, few works were devoted to electrical properties of $\mathrm{Ga}_{2} \mathrm{O}_{3}$ nanowires, and most of them are related to their gas sensing properties [10]. Recently, the field emission properties of $\mathrm{Ga}_{2} \mathrm{O}_{3}$ nanostructures with different morphologies such as nanowires, nanorods or cactus-like nanostructures have been reported [11-15]. In this work, we have studied the field emission properties of gallium oxide nanowires in different arrangements, namely aligned nanowires emerging from the lateral surfaces of microplates and random nanowires with high aspect ratio. The effect of Sn doping on the field emission behaviour has been also investigated.
The study of the morphology and field emission properties of all samples has been performed by means of a tuned scanning electron microscope. Characteristic curves of field emission current density versus applied electric field were recorded for different nanostructures and different vacuum gaps. The results show that gallium oxide nanowires have good field emission properties that are improved by Sn doping.

2 Experimental The synthesis route consists of a direct growth of gallium oxide nanostructures from either gallium oxide powders or metallic gallium as material sources on compacted gallium oxide pellets used as substrates. This method avoids the use of a foreign catalyst. The starting materials were placed into a tubular furnace and different arrangements of the nanostructures were synthesized after suitable thermal treatments. Figure 1 shows three representative morphologies of the micro- and nanostructures studied in this work, which depend on the precursor material and the thermal treatment used. Figure 1a shows undoped $\mathrm{Ga}_{2} \mathrm{O}_{3}$ microstructures obtained after a thermal treatment at $1500^{\circ} \mathrm{C}$ of compacted gallium oxide powders as source material [16]. Figure $1 b$ shows the results after a temperature profile with two plateaux at 1500 and $1350{ }^{\circ} \mathrm{C}$, which led to emerging parallel needles from preferential faces in the microstructures [17]. 

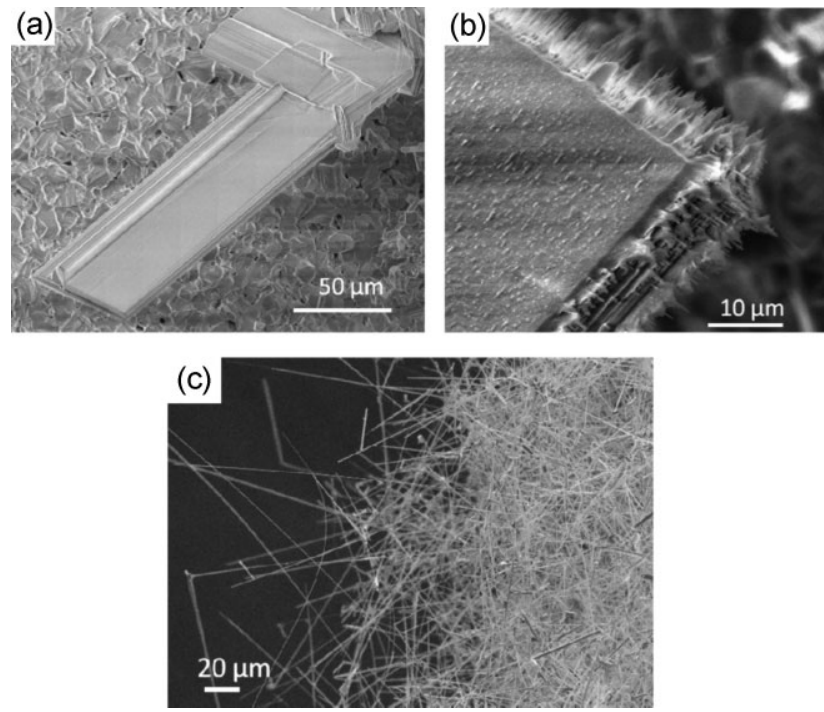

Figure 1 Representative SEM images of the $\mathrm{Ga}_{2} \mathrm{O}_{3}$ microstructures tested for field emission properties in the scanning electron microscope chamber. Undoped microstructures from gallium oxide powders obtained after (a) a single step temperature treatment and (b) a two-step profile. (c) Sn doped $\mathrm{Ga}_{2} \mathrm{O}_{3}$ nanowires obtained from pure metallic Ga.

On the other hand, pure $\mathrm{Ga}$ and tin oxide powders as precursor materials led to a high amount of Sn doped nanowires (Fig. 1c). In this last case, the thermal treatment was a two-step profile, with a first temperature plateau at $1150^{\circ} \mathrm{C}$ for $3 \mathrm{~h}$ followed by a second one at $1500^{\circ} \mathrm{C}$ for $15 \mathrm{~h}$. Morphology and field emission properties of the obtained structures have been studied in a scanning electron microscope (FEI Inspect S50). A special setup for field emission measurements has been designed and implemented into the SEM chamber. Two vertical copper plates were mounted over the specimen holder; the sample with nanostructures was attached to one of them, which acted as cathode, and the other one was the anode. Keithley 6514 electrometer and Keithley 2400 source-meter instruments were used to record the emission current and as power supply, respectively. With this setup our system enables to monitor the anode-cathode distance through the specimen holder remote control during observation in SEM. X-ray photoelectron spectroscopy (XPS) measurements were performed at the Synchrotron Elettra Trieste facility (ESCA-microscopy beamline) to determine the chemical composition and to calculate the work function of individual gallium oxide nanostructures.

3 Results and discussion We studied the dependence on the morphology of the field emission properties of gallium oxide micro- and nanostructures. Variations on the morphology are caused by changing the source material used to grow the nanostructures.

Starting with pure gallium oxide compacted powders, planar microstructures with flat faces or comb-like shapes

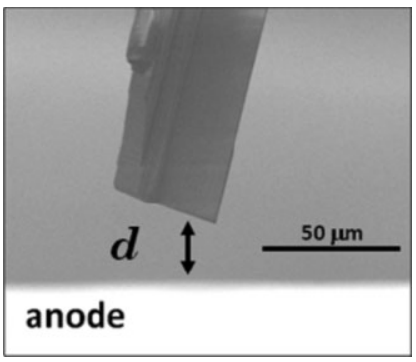

Figure 2 SEM image of a flat $\mathrm{Ga}_{2} \mathrm{O}_{3}$ microstructure placed at a distance ' $d$ ' of the anode electrode. The electrical current detected is just noise.

are developed depending on the thermal treatment. Figure 2 shows a secondary electron image of a flat $\mathrm{Ga}_{2} \mathrm{O}_{3}$ microplate at a distance ' $d$ ' from the anode, which can be controlled with the motorized specimen holder stage in the SEM chamber. This flat microstructure does not lead to significant electrical current, even at small ' $d$ ' distances. Undoped $\mathrm{Ga}_{2} \mathrm{O}_{3}$ can present, under appropriate growth conditions, electrical conductivity due to donors created by native oxygen vacancies, thus behaving as an n-type semiconductor [18]. However, in this case, the resistivity is still high and no electrical current has been detected. In semiconductors with rather low carrier concentration or electrical conductivity, the morphology influences drastically the field emission properties. Semiconductor nanowires are elongated structures suitable for field emission measurements due to the sharp tips with very low radii of curvature that enhance the field emission properties.

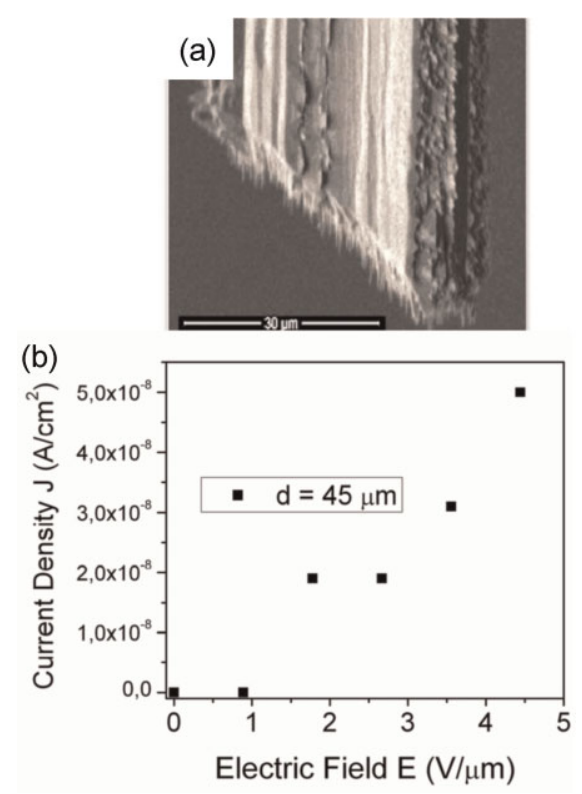

Figure 3 (a) SEM image of a $\mathrm{Ga}_{2} \mathrm{O}_{3}$ microstructure ended in nanoneedles. (b) Current density versus electric field curve for electrode distance of $45 \mu \mathrm{m}$. Although field emission current was recorded, it was not enough to determine turn on and threshold voltages. 
This is apparent in the case of the nanowire arrays emerging from lateral faces of some of the undoped planar. Figure 3a shows the SEM image of one of these microstructures with a set of parallel nanowires in one of its faces and Fig. $3 \mathrm{~b}$ shows the field emission current density measured on this face. The current densities were rather low for applied electric fields of up to $5 \mathrm{~V} / \mu \mathrm{m}$. This result shows that the presence of the parallel nanowires enhances the field emission of the flat microstructures. Although an increasing current density was detected as the electric field increased, its value remained below $1 \mu \mathrm{A} / \mathrm{cm}^{2}$ and the turn on electrical field for this structure could not be determined.

We have also studied the field emission behaviour of $\mathrm{Sn}$ doped nanowires. This dopant impurity introduces shallow donor levels and likely enhances electrical conductivity [19]. The above described synthesis route resulted in a high density of nanowires. Figure 4a shows the SEM image of the Sn doped nanowires, with the substrate pellet mounted on the cathode electrode and Fig. 4b shows a detail of the square marked in Fig. 4a. It can be observed that some of the nanowires are very long, in the range of $100 \mu \mathrm{m}$, so a very high aspect ratio is achieved. Figure $4 \mathrm{c}$ shows the field emission curves taken from this sample at electrode distances of 300 and $400 \mu \mathrm{m}$. We have applied a voltage of up to $200 \mathrm{~V}$ and the distance between electrodes is measured directly from SEM images. The fact that some nanowires are emerging about $100 \mu \mathrm{m}$ has been considered to calculate the electric field. To calculate the current density, the area of

(a)

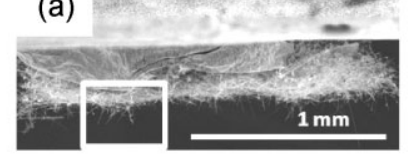

(b)

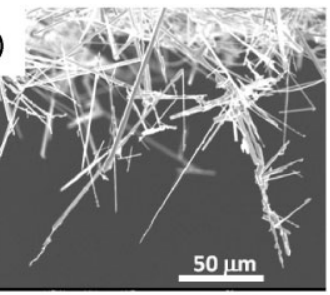

(c)

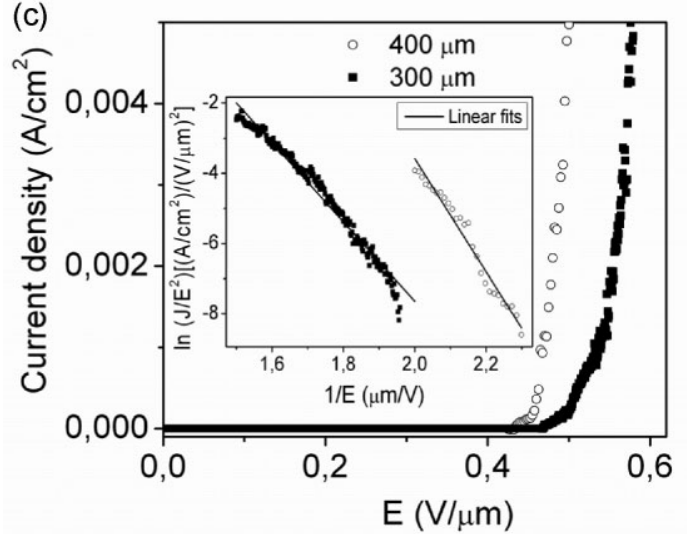

Figure 4 (a) SEM image of $\mathrm{Sn}$ doped $\mathrm{Ga}_{2} \mathrm{O}_{3}$ nanostructures mounted at the cathode. (b) Higher magnification SEM image of the marked square in (a). (c) Density current versus electric field curves for $d=300 \mu \mathrm{m}$ (black squares) and $d=400 \mu \mathrm{m}$ (open circles). Inset shows the linear fit of $\ln \left(J / E^{2}\right)$ versus $1 / E$. emerging electrons is also required. We have estimated this value from SEM images by measuring the area covered with nanostructures, as it can be seen in Fig. 4a. The results show that the threshold fields (corresponding to the current density of $1 \mathrm{~mA} / \mathrm{cm}^{2}$ ) were about 0.55 and $0.47 \mathrm{~V} / \mu \mathrm{m}$ for 400 and $500 \mu \mathrm{m}$ electrode distances, respectively. The field emission current-voltage characteristics are usually described in the framework of the Fowler-Nordheim $(\mathrm{F}-\mathrm{N})$ theory, in which the current density $J$ can be expressed as

$$
\begin{aligned}
& J=\left(\frac{A \beta^{2} E^{2}}{\phi}\right) \exp \left(-\frac{B \phi^{3 / 2}}{\beta E}\right), \\
& J=I / S\left(\mathrm{~mA} / \mathrm{cm}^{2}\right), \quad E=V / d(\mathrm{~V} / \mu \mathrm{m}),
\end{aligned}
$$

where $\phi$ is the work function of the emitters, $E$ the electric field and $\beta$ is the field enhancement factor caused by local geometry of the emitters. $A=1.54 \times 10^{-6} \mathrm{AeV} \mathrm{V}^{-2}$ and $B=6.83 \times 10^{3} \mathrm{eV}^{-3 / 2} \mathrm{~V} \mathrm{\mu m}^{-1}$ are universal constants. The F-N theory takes into account an enhancement factor, $\beta$, which is usually determined from the slope of the straight line obtained in the semi-logarithmic plot $\ln \left(J / E^{2}\right)$ versus $1 / E$ (see inset in Fig. 4c). The critical parameter in Eq. (1) to achieve a proper value for the enhancement factor is the work function of the emitter, $\phi$, in the exponential. Previous works regarding field emission properties of gallium oxide assumed $\phi$ estimated values between 4.15 and $4.8 \mathrm{eV}$ [12-14]. However, it is difficult to find accurate values of work functions energies for semiconductor oxide materials from experimental data. With this aim, we have performed XPS measurements of the $\mathrm{Sn}$ doped $\mathrm{Ga}_{2} \mathrm{O}_{3}$ nanostructures tested for field emission properties. Survey spectra obtained
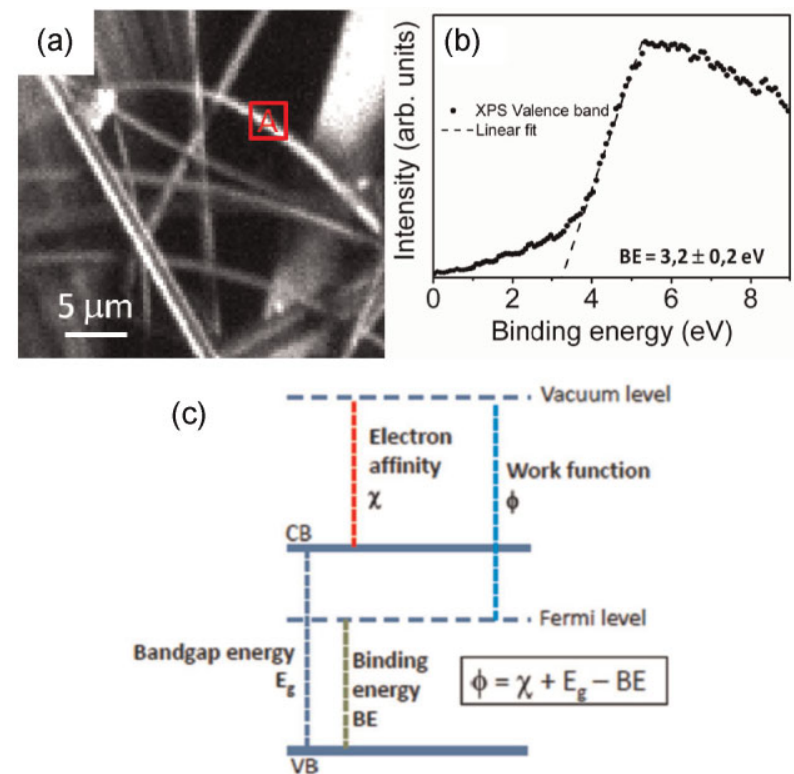

Figure 5 (online colour at: www.pss-a.com) (a) XPS image with the $\mathrm{Sn} 3 \mathrm{dBE}$ at $488 \mathrm{eV}$. (b) High resolution photoemission spectrum of the valence band recorded at the marked point of the XPS image. (c) Scheme of the energy levels in a semiconductor. 
from $\mathrm{Sn}$ doped $\mathrm{Ga}_{2} \mathrm{O}_{3}$ nanowires (not shown) present $\mathrm{Ga}$ (3d), O (1s) and Sn (3d) core level peaks. A XPS map obtained with the Sn 3d peak (binding energy; BE at $488 \mathrm{eV}$ ) is shown in Fig. 5a, which indicates that Sn concentration is rather homogenous in the nanowires. Furthermore, a representative high resolution photoemission spectrum of the valence band from the point marked in Fig. 5a is shown in Fig. 5b. This spectrum enabled us to determine the onset of the valence band, and accordingly with the scheme of Fig. 5c, $\phi=\chi+E_{\mathrm{g}}-\mathrm{BE}$, the work function of gallium oxide was calculated. The bandgap energy $\left(E_{\mathrm{g}}\right)$ is $4.9 \mathrm{eV}$, the electron affinity $(\chi)$ is $3.5 \mathrm{eV}$ [20] and the BE extrapolated from the line fitted to experimental data, shown in Fig. $4 \mathrm{~b}$ is $3.2 \pm 0.2 \mathrm{eV}$. Hence, the work function results $\phi=5.2 \pm 0.2 \mathrm{eV}$. The work function is known to depend on structural and chemical surface features. In fact, values from 4.5 to $5.4 \mathrm{eV}$ have been reported in multicomponent transparent conductive oxide films [21]. The increase of the value of $\phi$ measured in the $\mathrm{Sn}$ doped gallium oxide nanowires in comparison with data from bulk undoped $\mathrm{Ga}_{2} \mathrm{O}_{3}$ could be related to the presence of $\mathrm{Sn}$. With these values, the enhancement factors obtained for distance gaps of 300 and $400 \mu \mathrm{m}$ were 7000 and 3800, respectively.

A turn-on field of $1.97 \mathrm{~V} / \mu \mathrm{m}$, for $10 \mu \mathrm{A} / \mathrm{cm}^{2}$, and enhancement factor of 3287 was obtained in $\mathrm{In}_{2} \mathrm{O}_{3} / \mathrm{Ga}_{2} \mathrm{O}_{3}$ heterostructures for electrode distance of $400 \mu \mathrm{m}$ [6]. The authors claimed that their excellent emission behaviour was related to the high aspect ratio, good crystallinity and high conductivity of their heterostructures. Core-shell $\mathrm{Ga}_{2} \mathrm{O}_{3}-\mathrm{C}$ nanocables have also been demonstrated as good field emitters candidates [11]. The growth direction of the onedimensional $\mathrm{Ga}_{2} \mathrm{O}_{3}$ structures has also been proposed as a factor that influences the turn on field values [15]. Other materials with excellent field emission properties are carbon nanotubes, which on metal tips provide a threshold field of $0.7 \mathrm{~V} / \mu \mathrm{m}$ for an electrode distance of $0.7 \mathrm{~mm}$ [22]. In addition, field emission experiments performed in a SEM by using a sharp anode, suggested that emitters that contribute to the emitted current in large area measurements (similar to Fig. 4a) represent only a small proportion of exceptionally long and narrow carbon nanotubes [23]. The density of nanowires or nanotubes over rather large areas seems to be also a key factor for field emission applications as, for example, it has been demonstrated in $\mathrm{ZnO}$ nanowire arrays [5]. Our values - threshold field below $1 \mathrm{~V} / \mu \mathrm{m}$ and the field enhancement factor over 4000 , obtained for doped $\mathrm{Ga}_{2} \mathrm{O}_{3}$ nanowires are therefore excellent results for field emission applications. However, comparison with other reported results on field emission of nanowires is not straightforward. As described in Ref. [6] the there is a strong dependence of the turn on field on the distance between electrodes, and in the case of arrays of random nanowires, as the samples of this work, this distance is not exactly determined. The morphology of $\mathrm{Sn}$ doped $\mathrm{Ga}_{2} \mathrm{O}_{3}$ nanowires with a high density of wires in the cathode and with some very long wires emerging from it (see Fig. 4) may be the responsible for the obtained properties. These longer wires would be the main contributors to the measured emitted current. Hence, although further work is needed, these results suggest that Sn doped $\mathrm{Ga}_{2} \mathrm{O}_{3}$ nanowires are good candidates as cold emitters in future devices.

In summary, our results support the high performance of gallium oxide nanowires for field emission applications. Although undoped and planar microstructures did not show significant field emission current, Sn doped nanowires were demonstrated as a very good option as cold emitters. An experimental procedure was used to evaluate work function of $\mathrm{Ga}_{2} \mathrm{O}_{3}$ nanowires with the aid of XPS valence band spectroscopy. A special setup was mounted inside the SEM chamber to perform field emission measurements, which has enabled us to precisely control the distance between electrodes. The results showed very good values for the threshold electric field of large areas covered with $\mathrm{Sn}$ doped $\mathrm{Ga}_{2} \mathrm{O}_{3}$ nanowires, even better than in other tested materials.

Acknowledgements This work has been supported by MICINN (Projects MAT 2009-07882 and Consolider Ingenio CSD 2009-00013) and by BSCH-UCM (Project GR35-10A-910146). The authors are grateful to Dr Luca Gregoratti at the Sincrotrone Trieste for useful advises on XPS measurements.

\section{References}

[1] N. S. Xu and S. E. Huq, Mater. Sci. Eng. R 48, 47 (2005).

[2] J. Zhou, S. Z. Deng, N. S. Xu, J. Chen, and J. C. She, Appl. Phys. Lett. 83, 2653 (2003).

[3] J. Liu, Z. Zhang, Y. Zhao, X. Su, S. Liu, and E. Wang, Small 1, 310 (2003).

[4] Y. T. Lin, C. Y. Chen, C. P. Hsiung, K. W. Chang, and J. Y. Gan, Appl. Phys. Lett. 89, 063123 (2006).

[5] X. Wang, J. Zhou, C. Lao, J. Song, N. Xu, and Z. L. Wang, Adv. Mater. 19, 1627 (2007).

[6] J. Lin, Y. Huang, Y. Bando, C. Yang, C. Li, and D. Goldberg, ACS Nano 4, 2452 (2010).

[7] E. Nogales, J. A. García, B. Méndez, and J. Piqueras, J. Appl. Phys. 101, 033517 (2007).

[8] E. Nogales, B. Méndez, and J. Piqueras, Nanotechnology 19, 035713 (2008).

[9] E. Nogales, J. A. García, B. Méndez, and J. Piqueras, Appl. Phys. Lett. 91, 133108 (2007).

[10] Y. Huang, S. Yue, Z. Wang, Q. Wang, C. Shi, Z. Xu, X. D. Bai, C. Tang, and C. Gu, J. Phys. Chem. B 110, 796 (2006).

[11] J. Zhan, Y. Bando, J. Hu, Y. Li, and D. Goldberg, Chem. Mater. 16, 5158 (2004).

[12] Y. Huang, Z. Wang, Q. Wang, C. Gu, C. Tang, Y. Bando, and D. Goldberg, J. Phys. Chem. C 113, 1980 (2009).

[13] C. Cao, Z. Chen, X. An, and H. Zhu, J. Phys. Chem. C 112, 95 (2008).

[14] Y. Bayam, V. J. Logeeswaran, A. M. Katzenmeyer, R. J. Chacon, M. C. Wong, C. E. Hunt, and M. Saif Islam, Proceedings 8th IEEE Conference on Nanotechnology (IEEE, Piscataway, NJ, USA, 2008), pp. 573-575.

[15] G. Sinha, A. Datta, S. K. Panda, P. G. Chavan, M. A. More, D. S. Joag, and A. Patra, J. Phys. D 42, 185409 (2009). 
[16] E. Nogales, B. Méndez, and J. Piqueras, Appl. Phys. Lett. 86, 113112 (2005).

[17] E. Nogales, B. Méndez, J. Piqueras, and J. A. García, Nanotechnology 21, 115201 (2009).

[18] L. Binet and D. Gourier, J. Phys. Chem. Solids 59, 1241 (1998).

[19] M. Orita, H. Ohta, M. Hirano, and H. Hosono, Appl. Phys. Lett. 77, 4166 (2000).
[20] T. Minami, T. Miyata, and T. Yamamoto, Surf. Coat. Technol. 108-109, 583 (1998).

[21] J. Robertson and B. Falabretti, Mater. Sci. Eng. B 135, 267 (2006).

[22] D. Y. Zhong, G. Y. Zhang, S. Liu, T. Sakurai, and E. G. Wang, Appl. Phys. Lett. 80, 506 (2002).

[23] J. M. Bonard, K. A. Dean, B. F. Coll, and C. Klinke, Phys. Rev. Lett. 89, 197602 (2002). 